\title{
MODELO TEÓRICO DE INDICADORES DE RESPONSABILIDAD SOCIAL EN SALUD
}

THEORETICAL MODEL OF INDICATORS OF SOCIAL RESPONSIBILITY IN HEALTH

Danny Amado Gómez Perdomo 1

Resumen

\begin{abstract}
Con el propósito de realizar un análisis reflexivo del grado de cumplimiento de las políticas de Responsabilidad Social en el Sector Salud, se consideran los siguientes aspectos: dimensión social, dimensión económica y dimensión medio ambiental, promovidos por la UNESCO en concordancia con la OMS y la declaración de salud pública como derechos fundamentales humanos. Para la construcción de los indicadores se realizó revisión y análisis documental del estado del arte y el contexto; de principios, criterios y descriptores que constituyen la responsabilidad social de acuerdo con la Declaración Universal sobre Bioética y Derechos Humanos de la UNESCO que incluye la responsabilidad social como principio de la bioética y la salud pública.
\end{abstract}

Palabras Claves: Responsabilidad Social; Salud Pública; Indicadores de Responsabilidad Social.

Abstract

In order to carry out a reflexive analysis of the degree of compliance with Social Responsibility policies in the Health field, the following aspects are considered: social dimension, economic dimension and environmental dimension, promoted by UNESCO in accordance with WHO and the declaration of public health as fundamental human rights. For the construction of the indicators, a review and analysis of the state of the art and the context were carried out; of principles, criteria and descriptors that constitute social responsibility in accordance with UNESCO's Universal Declaration on Bioethics and Human Rights that includes social responsibility as a principle of bioethics and public health.

Keywords: Social Responsibility; Public Health; Social Responsibility Indicators.

\section{Introducción}

La relación de la responsabilidad social y la salud pública se puede expresar sinérgicamente, una vez se tenga claro las consideraciones que las definen. Para esto, se comienza con explicar cómo concibe la Organización Mundial de la Salud

\footnotetext{
${ }^{1}$ Licenciado en Contaduría Pública, Magíster Scientiarum en Auditoria. Académico de la Escuela de Administración y Auditoría de la Facultad de Ciencias Sociales y Económicas de la Universidad Católica del Maule. E-mail: dgomez@ucm.cl
} 
(OMS) la responsabilidad social, como la obligación de dirigir las actividades educativas, investigadoras y de servicio a atender las necesidades prioritarias de la comunidad, región y/o nación (OMS, 2015).

A través de la implementación de un sistema integral de garantía de la calidad, que permita ofrecer a los usuarios y sus familias (Rusinque y Constanza 2016) servicios de salud enmarcados en la calidad, la seguridad, la humanización de la atención, como un modelo de gestión orientado a la excelencia a través del compromiso ético (Sánchez, Casabella, y Trench, 2009).

Es por lo anterior que el concepto de responsabilidad social se visualiza como una herramienta que debe estar presente en las organizaciones prestadoras de salud con el fin de atender a la necesidad de salud y bienestar de la sociedad; así, idealmente sería un compromiso directo, el cual debe provenir del direccionamiento estratégico de las organizaciones de salud hasta la gerencia de los procesos diarios que se llevan a cabo en los distintos niveles de cargo y de sus respectivos colaboradores.

Con este enfoque, se han incorporado objetivos sociales, medioambientales y económicos a los objetivos tradicionales del servicio de salud pública precisamente, estos conceptos como dimensión en la estrategia de responsabilidad social tiene claras concordancias con las disciplinas de calidad, y caracterización de los descriptores de la salud pública como derechos fundamentales humanos.

En este trabajo se pretende presentar un conjunto de indicadores de responsabilidad social que las instituciones de salud puedan aplicar para medir el desarrollo y avances alcanzados en la gestión ética y socialmente responsable, y que a la par sirvan para ganar una mayor confianza de los usuarios sobre los servicios de salud, pues las demandas de los pacientes ya no se basan únicamente en la calidad de la atención médica, sino que cada vez valoran más la actuación de la institución en términos de responsabilidad frente al entorno y la sociedad.

La responsabilidad social se ha convertido en una práctica corporativa esencial en la mayoría de las organizaciones. El concepto principal de ser socialmente responsable de acuerdo a Hammadeh y Mohamad (2016), es asegurar que las industrias y organizaciones participen en operaciones éticas de una manera que beneficie comunidades circundantes.

La responsabilidad social ha atraído una atención significativa en varias organizaciones desde mediados del siglo veinte. En la actualidad es un sin precedentes de prácticas de compromiso corporativo que busca mejorar la relación entre gerentes, empleados y el entorno. Ser socialmente responsable significa que una organización está comprometida con sus operaciones sociales 
para garantizar crecimiento sostenible, desarrollo y mejora de las condiciones sociales del medio donde se desarrolla (CCE, 2001).

Sin embargo, más allá de establecer un debate ético acerca de la importancia de que las organizaciones reconozcan su responsabilidad social, a esta se le ha respetado históricamente su carácter "voluntario", el cual no debe entrar en contradicción con el hecho de que para que se logren resultados efectivos, debe ser gestionada coherentemente al interior de la organización. Se reconoce, entonces, que la responsabilidad social es un estilo de ser de la institución y de cada una de las personas que la integran (...) Es algo que debe ser gestionado porque trata de establecer normas y procedimientos que sirvan para movilizar y encauzar la sensibilidad social de cada uno de los empleados (Terán et al., 2017).

Los agresivos cambios del entorno, conjuntamente con los avances tecnológicos, la evolución de estudios científicos y la concepción de la responsabilidad social en los últimos años, presentan un gran cambio. Este contexto cada vez es más generalizado, afirma Soriano Álvarez (2015), a partir de las expectativas de globalización y unificación de criterios que se buscan para alcanzar que el ser humano construya para sí mismo y la sociedad en la que vive un estándar de calidad de vida en un estado completo de salud, tal y como la concibe la Organización Mundial de la Salud (OMS).

El enfoque del compromiso social de una institución de salud, según Boelen (2015), se caracteriza por su triple capacidad: de identificar problemas de salud actuales y futuros, de implementar estrategias que los solucione y de manifestar un impacto real, de manera que el compromiso social es el más alto nivel de obligación social en comparación con lo que comúnmente se llama de responsabilidad social o de respuesta social. Una institución socialmente responsable es consciente de las necesidades prioritarias de la sociedad y se rige principalmente por la intuición y el sentido común, mientras que una institución socialmente comprometida tiene un conocimiento explícito de las necesidades por medio de una evaluación crítica de los hechos y, por lo tanto, se encuentra en una mejor posición para dirigir sus planes de acción y sus recursos a los objetivos claramente identificados para obtener resultados medibles.

En este mismo orden de ideas, se vuelve relevante la simbiosis entre el compromiso social y la responsabilidad social, a través de una ética que precede toda actuación socialmente responsable y socialmente comprometida. Es por ello que ser ético excede ser legal; cuando las organizaciones enfatizan la prevención de conductas ilegales, incrementando la vigilancia y el control, sólo desarrollan una estrategia que tiende al acatamiento. Para la formación del êthos se requiere una estrategia de integridad que lleve a los miembros de la organización al convencimiento profundo de ajustar su conducta a una serie de valores éticos. Gilli (2006) sostiene que la internalización de valores éticos en los actores de una 
organización hace prescindible en cierta medida el uso de controles externos, no en igual medida controles internos.

El término "responsabilidad social", en general, encuentra su actual marco de referencia en las obligaciones de las instituciones, especialmente las empresas, no sólo para con sus directivos, sino para con todos los implicados en sus actividades llamados stakeholders (trabajadores, proveedores, clientes, comunidad y directivos) y para con el medioambiente.

En este sentido el Libro Verde (CCE, 2001) manifiesta que "ser socialmente responsable no significa solamente cumplir plenamente las obligaciones jurídicas, sino también ir más allá de su cumplimiento invirtiendo en el capital humano, el entorno y las relaciones con los interlocutores" (p. 4). Los cuales pueden ser aglutinados en una dimensión interna y otra dimensión externa.

Dimensión interna: esta dimensión presenta el bienestar social en los trabajadores de la organización, tanto en la inversión de los recursos humanos, la salud y la seguridad; y en las prácticas medioambientales la gestión de los recursos naturales utilizados en la producción (CCE, 2001). Por lo tanto, son las decisiones y acciones habituales de funcionamiento de una organización.

En cuanto a la dimensión externa: lejos de circunscribirse al perímetro de las empresas, la responsabilidad social se extiende hasta las comunidades locales e incluye, además de a los trabajadores y directivos, a un amplio abanico de interlocutores externos, como defensores de los intereses de las comunidades locales, regionales y el medio ambiente en contexto (CCE, 2001).

\section{Consideración de los principios de Responsabilidad Social}

En referencia a los principios de responsablidad social, argumentan Argandoña y Silva (2011) la idea de que una organización adopte una conducta socialmente responsable surge de la necesidad de su contribución al desarrollo sostenible del entorno y la comunidad. Aunque no pretende dar una lista definitiva de principios de responsabilidad social, la ISO 26000 aconseja adoptar, como mínimo, estos siete, a fin de facilitar la integración de la responsabilidad social en toda la organización (p. 5). 


\section{Tabla1. Principios de la Responsabilidad Social}

\begin{tabular}{|c|c|}
\hline Rendición de cuentas & $\begin{array}{l}\text { Se invita a la organización a rendir cuentas por los impactos } \\
\text { económicos, sociales y ambientales de su actuación, lo cual } \\
\text { también implica asumir responsabilidad por sus impactos negativos } \\
\text { y el compromiso de tomar las medidas pertinentes para repararlos } \\
\text { y evitar repetirlos. También invita a las organizaciones a aceptar el } \\
\text { escrutinio público de sus actividades y a asumir un papel activo en } \\
\text { responder a dicho escrutinio. }\end{array}$ \\
\hline Transparencia & $\begin{array}{l}\text { Se invita a las organizaciones ser transparentes en aquellas } \\
\text { actividades que desarrollan y afectan a la sociedad y al medio } \\
\text { ambiente. De este modo, sugiere que la organización debería } \\
\text { suministrar toda la información que requieran las partes } \\
\text { interesadas, en un lenguaje accesible e inteligible. El principio } \\
\text { excluye, sin embargo, que se publique la información protegida por } \\
\text { la propiedad intelectual o la que pueda causar incumplimientos de } \\
\text { obligaciones legales. }\end{array}$ \\
\hline $\begin{array}{l}\text { Comportamiento } \\
\text { Ético }\end{array}$ & $\begin{array}{l}\text { Se invita a tener un impacto verdaderamente positivo en el } \\
\text { desarrollo sostenible, la organización debería regirse por criterios } \\
\text { de honestidad, equidad e integridad, lo que significa que la } \\
\text { empresa no debería perseguir únicamente el beneficio económico, } \\
\text { sino también tratar de maximizar los impactos positivos en su } \\
\text { entorno social y medioambiental, y minimizar los negativos. }\end{array}$ \\
\hline $\begin{array}{l}\text { Respeto a los } \\
\text { intereses de las partes } \\
\text { interesadas }\end{array}$ & $\begin{array}{l}\text { Señala que la organización debería respetar y atender los intereses } \\
\text { y requerimientos de las partes interesadas. Esto es así porque, aun } \\
\text { cuando los objetivos de una empresa puedan circunscribirse a los } \\
\text { intereses de sus dueños, existe un conjunto de actores o partes } \\
\text { interesadas que, si bien no forman parte de la empresa, tienen } \\
\text { unas necesidades y unos intereses legítimos que pueden verse } \\
\text { afectados por las actividades de la empresa. Se recomienda tener } \\
\text { en cuenta a estos grupos de interés a la hora de operar y tomar } \\
\text { decisiones. }\end{array}$ \\
\hline $\begin{array}{l}\text { Respeto al principio } \\
\text { de legalidad }\end{array}$ & $\begin{array}{l}\text { Aconseja respetar el principio de legalidad o supremacía del } \\
\text { derecho, lo que pasa por reconocer que ningún individuo u } \\
\text { organización tiene la potestad de actuar fuera de la ley. En el } \\
\text { ámbito de la RS, el respeto al principio de legalidad significa que } \\
\text { la organización debería respetar y cumplir las leyes y regulaciones } \\
\text { aplicables y, por tanto, debería tomar las medidas necesarias para } \\
\text { estar al corriente y cumplir la legislación vigente en materia de RS. }\end{array}$ \\
\hline
\end{tabular}




\begin{tabular}{|l|l|}
\hline $\begin{array}{l}\text { Respeto a la } \\
\text { normativa } \\
\text { internacional de } \\
\text { comportamiento }\end{array}$ & $\begin{array}{l}\text { Se invita respetar la normativa internacional de comportamiento } \\
\text { las salvaguardas sociales y medioambientales. Y para el caso de } \\
\text { que la ley de su jurisdicción entre en colisión con la normativa } \\
\text { internacional, la organización debería revisar la naturaleza de sus } \\
\text { relaciones y actividades en esa jurisdicción y evitar ser cómplice } \\
\text { de comportamientos que no sean compatibles con la normativa } \\
\text { internacional de RS. }\end{array}$ \\
\hline $\begin{array}{l}\text { Se invita a que la organización debería respetar los derechos } \\
\text { humanos, así como reconocer su importancia y universalidad, es } \\
\text { derechos humanos } \\
\text { decir, que estos derechos son aplicables a todos los individuos } \\
\text { de todos los países y culturas. Y, en el caso de que los derechos } \\
\text { humanos no sean garantizados en su ámbito de actuación, bien } \\
\text { sea por un vacío legal o por prácticas inadecuadas, la organización } \\
\text { debería hacer todo lo que esté a su alcance para respetar y } \\
\text { proteger esos derechos. }\end{array}$ \\
\hline
\end{tabular}

Fuente: Argandoña y Silva (2011)

\section{La Salud Pública}

Alo largo de lasúltimas décadas se ha intentado establecer una conceptualización estándar que pueda definir en todo su contexto la salud pública y su actuación en la moderna sociedad.

La salud pública está considerada como una rama de la medicina y a su vez como una disciplina de la ciencia política encargada de la protección de la salud a nivel poblacional. En este sentido, afirman Kottow y Schramm (2001), busca mejorar las condiciones de salud de las comunidades mediante la promoción de estilos de vida saludables, las campañas de c, oncienciación, la educación y la investigación.

Así mismo expresa las necesidades de atención médica cuyo propósito central es iluminar el sentido de cuidado de la salud que resulta "especial" de acuerdo a Daniels (1983), y debe tratarse de manera diferente a otros servicios sociales. Específicamente, incluso en sociedades en las que la gente tolera las desigualdades significativas y generalizadas en la distribución de la mayoría de los bienes sociales, muchos sienten que existen razones especiales de justicia para distribuir la asistencia sanitaria de manera más equitativa. 
Algunos estudiosos del área presentan sus aportes más relevantes a continuación:

\section{Tabla 2. Salud Pública}

\begin{tabular}{|l|l|}
\hline UNESCO (2005) & $\begin{array}{l}\text { La promoción de la salud y el desarrollo social para sus pueblos es } \\
\text { un cometido esencial de los gobiernos, que comparten todos los } \\
\text { sectores de la sociedad. }\end{array}$ \\
\hline Pérez (2002) & $\begin{array}{l}\text { La Salud Pública es la práctica social integrada que tiene como sujeto } \\
\text { y objeto de estudio la salud de las poblaciones humanas, y se le } \\
\text { considera como la ciencia encargada de prevenir la enfermedad, la } \\
\text { discapacidad, prolongar la vida, fomentar la salud física y mental, } \\
\text { mediante los esfuerzos organizados de la comunidad, para el } \\
\text { saneamiento del ambiente y desarrollo de la maquinaria social, } \\
\text { para afrontar los problemas de salud y mantener un nivel de vida } \\
\text { adecuado. }\end{array}$ \\
\hline Navarro (1998) & $\begin{array}{l}\text { Es una actividad científica encaminada a mejorar la salud de la } \\
\text { población. }\end{array}$ \\
\hline Terris (1992) & $\begin{array}{l}\text { Acción científica de prevenir las enfermedades, prolongar la vida, } \\
\text { fomentar la salud y la eficiencia física y mental, mediante el esfuerzo } \\
\text { organizado de la comunidad para: el saneamiento del medio } \\
\text { ambiente; el control de las enfermedades transmisibles; la educación } \\
\text { sanitaria; la organización de los servicios médicos y de enfermería; y } \\
\text { el desarrollo de los mecanismos sociales que aseguren al individuo } \\
\text { y a la comunidad un nivel de vida adecuado para la conservación de } \\
\text { la salud. }\end{array}$ \\
\hline Salud (OMS 1984)
\end{tabular}

Fuente: Elaboración propia.

Los principios y valores éticos son de extrema importancia y ampliamente compartidos involucrados tanto en el diseño como en los debates sobre la responsabilidad social ante los sistemas de salud (Brock, 1994).

Considerando que la UNESCO, conforme a su constitución en 1945, ha de desempeñar un papel en la definición de principios universales basados en valores éticos comunes que orienten los adelantos científicos y el desarrollo tecnológico y la transformación social, a fin de determinar los desafíos que surgen en el ámbito de la ciencia y la tecnología teniendo en cuenta la responsabilidad de las generaciones actuales para con las generaciones venideras, y que las cuestiones de bioética, que forzosamente tienen una dimensión internacional, se deben 
tratar como un todo, basándose en los principios ya establecidos en la Declaración Universal sobre el Genoma Humano y los Derechos Humanos y la Declaración Internacional sobre los Datos Genéticos Humanos, y teniendo en cuenta no sólo el contexto científico actual, sino también su evolución futura (Brussino, 2015).

En circunspección de la aserción que establece la Organización Mundial de la Salud, OMS, en función de la Salud Pública, y la categorización de los derechos fundamentales de todo ser humano, se promueve la Declaración Universal sobre Bioética y Derechos Humanos por parte de la UNESCO para octubre de 2005, donde contiene, en su artículo 14, fundamentos para la Responsabilidad social y la salud.

Bergel (2007) afirma que los objetivos de esta declaracion universal pretende como marco de acción “...promover el respeto de la dignidad humana y proteger los dechos humanos y las libertados fundamentales de conformidad con el derecho internacional de los derechos humanos" (p. 13).

La promoción de la salud y el desarrollo social para sus pueblos es un cometido esencial de los gobiernos, que comparten todos los sectores de la sociedad (UNESCO, 2005).

Teniendo en cuenta que el goce del grado máximo de salud que se pueda lograr es uno de los derechos fundamentales de todo ser humano sin distinción de raza, religión, ideología política o condición económica o social (Bergel, 2007), los progresos de la ciencia y la tecnología deberían fomentar las siguientes consideraciones de responsabilidad social: 


\section{Tabla 3. Responsabilidad Social y Salud}

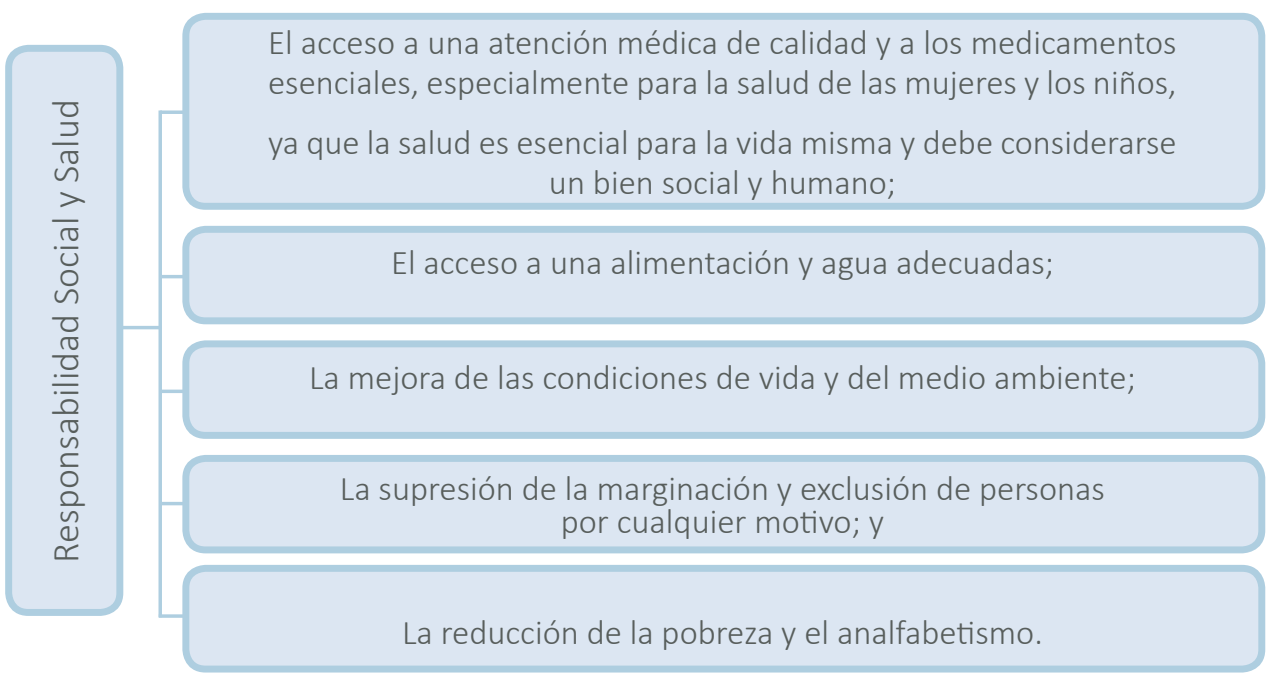

Fuente: Artículo 14, Declaración Universal sobre Bioética y Derechos Humanos por parte de la UNESCO (2005).

En virtud de esto, la atención medica de calidad no puede desentenderse de una respuesta adecuada a los condicionantes económicos y sociales de la salud, en consecuencia, la salud en su función de disfrute de los derechos humanos tiene como requerimientos la paz, la educación, la justicia social y la equidad. Por ende, la responsabilidad social en servicios de medicina ha de cumplir a cabalidad su objetivo de evitar las desigualdades socioeconómicas en la distribución de los recursos escasos de la salud y los riesgos en su aplicación (Correa y Valencia, 2016).

Considerando lo anteriormente expuesto, se da la premisa a la formulación e implementación de los indicadores de responsabilidad social que puedan medir los resultados de los planes de acción ejecutados en virtud del compromiso y responsabilidad social dentro del sector de salud pública.

En la actualidad no hay un general consenso sobre la definición exacta de la responsabilidad social dentro del sector de la salud. El término, por su característica polisémica (Lozano y Soler, 2000), se usa comúnmente para denotar un modo de crear valor comercial y compromiso con un objetivo de cumplir con las expectativas sociales públicas, éticas y legales.

Simplemente indicando, la responsabilidad social se refiere a una manera en que una institución de la salud utilizará sus recursos en esfuerzos para beneficiar a la sociedad desfavorecida (Hammadeh y Mohamad, 2016). Según el World 
Business Council (2017), la responsabilidad social en salud toma en cuenta el compromiso de garantizar que la sociedad, la familia, empleados, y la comunidad más grande se beneficie de las instalaciones de salud cercanas de su localidad.

Otros eruditos, como Benomram (2010), han dicho que la responsabilidad social se ocupa principalmente de establecer programas, prácticas y políticas que se incorporan en toma de decisiones empresariales y operaciones con el objetivo de asegurar que una organización maximice sus impactos beneficiosos en la sociedad; así mismo, afirma que en el sector de la salud, la responsabilidad social no tiene la implementación sistemática y plenamente lograda, porque existe un número de retos pendientes con respecto a la prestación de servicios, además de la participación de la comunidad, unidos con una serie de factores que también contribuyen a la baja implementación de responsabilidad social en las prácticas de la salud.

La salud es un factor que se encuentra arraigado en el marco constitucional y legal como un derecho fundamental humano de tipo individual y colectivo. En razón a este argumento que hacen Rusinque y Constanza (2016), adquiere una gran importancia para las implementación de modelos de desarrollo social del estado buscando garantizar la mejor calidad de vida posible para todas las personas en aras de conservar altos niveles de bienestar social y formas de vida saludables indiferentemente del nivel económico-social de las personas.

Los indicadores de gestión, según Cardona y Giraldo (2010), son la única medida objetiva que tienen las organizaciones para realizar evaluación de cada una de sus actividades desde su objeto social, por ello la aplicación de los indicadores les permitirá identificar cuál es el rol social de la organización y el impacto que le brinda a sus colabores y entorno, sin descuidar cada uno de los ejes de desarrollo, considerando cuales aplican de acuerdo a la naturaleza y procesos de la organización.

\section{Indicadores de Responsabilidad Social}

Muchos investigadores están en la búsqueda de indicadores que clasifiquen una organización como socialmente responsable. Conforme a Ortiz y Palavecino (2005) los denominados indicadores de responsabilidad social tienen una tendencia entre los inversores a comprobar si las organizaciones donde invierten tienen en cuenta estos parámetros. Como consecuencia, se está comenzando a mover una masa crítica que induce a las instituciones a responder cuestionamientos éticos exigidos por inversores que se enrolan entre lo socialmente responsable (Chicharro, 2006). 
Sea cual sea la estrategia que se adopte, para regir las prácticas de responsabilidad social deberá basarse en unos criterios que marquen las directrices principales a seguir (Sánchez et al., 2009).

Se resumen los criterios definidos por las UNESCO en congruencia con lo establecido por la OMS, como principios guías de indicadores de responsabilidad social para la salud.

Tabla 4. Responsabilidad Social, dimensiones y criterios

\begin{tabular}{|l|l|}
\hline \multicolumn{1}{|c|}{ Dimensión } & \multicolumn{1}{c|}{ Criterio } \\
\hline Social & $\begin{array}{l}\text { El acceso a una atención médica de calidad y a los } \\
\text { medicamentos esenciales, especialmente para la } \\
\text { salud de las mujeres y los niños, ya que la salud es } \\
\text { esencial para la vida misma y debe considerarse un } \\
\text { bien social y humano. }\end{array}$ \\
\hline Medio Ambiental & El acceso a una alimentación y agua adecuadas. \\
\hline Económico & $\begin{array}{l}\text { La mejora de las condiciones de vida y del medio } \\
\text { ambiente; }\end{array}$ \\
\hline Económico & $\begin{array}{l}\text { La supresión de la marginación y exclusión de } \\
\text { personas por cualquier motivo; y }\end{array}$ \\
\hline Social & Económico \\
Medio Ambiental & La reducción de la pobreza y el analfabetismo. \\
\hline Social & Económico
\end{tabular}

Fuente: Elaboración Propia.

Cada uno de los criterios lleva asociados un conjunto de indicadores de primer nivel. 
Tabla 5. Responsabilidad Social, Indicadores

\begin{tabular}{|c|c|}
\hline Dimensión & Indicadores \\
\hline Social & $\begin{array}{l}\text { 1) Tasa de manejo integral en Salud en los sectores } \\
\text { menos favorecidos } \\
\text { 2) Tasa de acceso al servicio de Salud por nivel de } \\
\text { ingreso de los pacientes. } \\
\text { 3) Tasa de buena salud general. } \\
\text { a) Tasa de buena salud para los jóvenes. } \\
\text { b) Tasa de envejecimiento en buena salud. } \\
\text { c) Tasa de mejoras de la salud mental. } \\
\text { d) Tasa de disminución de enfermedades } \\
\text { transmisibles. } \\
\text { e) Tasa de disminución de las enfermedades no } \\
\text { transmisibles. } \\
\text { 6) Tasa de participación en la creación de espacios o } \\
\text { escenarios favorables para la Salud. } \\
\text { o) Tasa de participación en la creación de espacios } \\
\text { multisectorial en relación con la Salud. } \\
\text { 4) Tasa de disminución de las lesiones derivadas de } \\
\text { actos violentos. } \\
\text { a) Tasa de disminución de las lesiones derivadas de } \\
\text { accidentes. } \\
\text { 5) Desarrollo de planes de acción en salud para un } \\
\text { entorno físico. } \\
\text { a) Desarrollo de planes de acción en salud para un } \\
\text { b) Desarrollo de planes de acción en salud para un } \\
\text { a) }\end{array}$ \\
\hline
\end{tabular}




\begin{tabular}{|c|c|}
\hline Económico & $\begin{array}{l}\text { 1) Tasa de eliminación progresiva de las limitaciones } \\
\text { financieras. } \\
\text { 2) Cantidad de planes de inversión en salud. } \\
\text { 3) Tasa de formación de alianzas y redes de expertos } \\
\text { para brindar respaldo a la planificación de las } \\
\text { actividades. } \\
\text { a) Tasa de mantenimiento de alianzas y redes de } \\
\text { expertos para brindar respaldo a la planificación } \\
\text { de las actividades. } \\
\text { b) Tasa de formación de alianzas y redes de } \\
\text { expertos para brindar respaldo a la ejecución de } \\
\text { las actividades. } \\
\text { c) Tasa de mantenimiento de alianzas y redes de } \\
\text { expertos para brindar respaldo a la ejecución de } \\
\text { las actividades. } \\
\text { 4) Tasa de implementación estratégica de desarrollo de } \\
\text { políticas de aspectos económicos. }\end{array}$ \\
\hline Medio Ambiente & $\begin{array}{l}\text { 1) Tasa de compromiso con el desarrollo continuo del } \\
\text { sistema de gestión Ambiental. } \\
\text { 2) Tasa de uso eficiente de los recursos naturales. } \\
\text { a) Tasa de reducción de emisiones y producción de } \\
\text { desperdicios contaminantes. } \\
\text { 3) Tasa de desarrollo de una política de gestión de } \\
\text { riesgos sensata en el uso de productos químicos } \\
\text { contaminantes. } \\
\text { 4) Tasa de cumplimiento de la reglamentación nacional } \\
\text { e internacional en materia de protección al medio } \\
\text { ambiente. }\end{array}$ \\
\hline
\end{tabular}

Fuente: Elaboración Propia. 


\section{Conclusiones}

El importante trato social, humano y manejo económico que se deriva de la atención en salud, hace que la responsabilidad social para la salud pública adquiera un enorme protagonismo en el marco socio-económico y político en el que se desenvuelven estas instituciones y mantiene esta materia en un estado de armonía y permanente evolución.

La salud pública según la definición de la Organización Mundial de la Salud es el estado completo de bienestar físico, mental y social, entonces el actuar sobre el espectro de las determinantes políticas, económicas, culturales, ambientales y de salud debe ser imprescindible. Consecuentemente, y en concordancia con lo expuesto por Boelen (2015), los principales grupos interesados, como son los entes formuladores de políticas, las asociaciones profesionales, las organizaciones de servicios de salud, las empresas de seguros de salud, las instituciones académicas, las industrias y la sociedad civil en general, deben trabajar de forma sinérgica garantizando el goce de los valores básicos de calidad, equidad, relevancia y rentabilidad relacionados con el cuidado en salud.

La salud pública en su función de disfrute de los derechos humanos, y de acuerdo a lo que sostienen Correa y Valencia (2016), tiene como requerimientos la paz, la educación, la justicia social y la equidad. En consecuencia, la responsabilidad social en la salud ha de cumplir a cabalidad su objetivo de evitar las desigualdades socioeconómicas en la distribución de los recursos escasos de la salud y los riesgos en su aplicación.

Una organización que argumente que es socialmente responsable debe estar en condiciones de presentar con transparencia los resultados de su evaluación de operaciones en los cuales se evidencie que no está afectando de manera negativa a los grupos de interés propios y que, por el contrario, tal como afirma Chicharro (2006) está actuando para lograr el mejoramiento continuo en su gestión frente a ellos y el entorno.

Es conveniente elaborar nuevos enfoques, nuevos criterios, nuevas acciones de la responsabilidad social para garantizar que el progreso de la salud pública, la ciencia y la tecnología contribuya a la justicia, a la equidad, la mitigación y erradicación de las barreras de acceso al sistema de salud, y sirva al interés de la humanidad. 


\section{Referencias}

Argandoña, A., y. Silva, I. (2011). ISO 26000, una guía para la responsabilidad social de las organizaciones. Business School Universidad de Navarra.

Benomran, F. (2010). Medical responsibility in the United Arab Emirates. Journal Forensic Legal Medicine, 17, 188-193.

Bergel, S. D. (2007). Responsabilidad social y salud. Revista Latinoamericana de Bioética, 7(12), 10-27.

Boelen, C. (2015). Improving the Impact on Health: The Social Accountability Approach. Texto \& Contexto-Enfermagem, 24(3), 615-616.

Brock, D. W. (1994). Ethical foundations of the Clinton administration's proposed health care system. JAMA, 271(15), 1189-1196.

Brussino, S. (2015). Responsabilidad ética y social de los profesionales en el contexto de la investigación universitaria en salud. Cuadernos de Ética, 28, 28-41.

Cardona, C., y Giraldo, L. (2010). Estandarización de indicadores de responsabilidad social empresarial propuestas por organizaciones de reconocimiento mundial. Administración del Desarrollo Humano y Organizacional. Universidad Tecnológica de Pereira.

CCE. (2001). Libro verde: fomentar un marco europeo para la responsabilidad social de las empresas. Comisión de las Comunidades Europeas.

Chicharro, M. (2006). Propuesta de indicadores de responsabilidad social propuesta de indicadores de responsabilidad social. Revista Universo Contábil, 2(1), 90102.

Correa, A., y Valencia, A. (2016). Responsabilidad social y ética medico sanitaria. Ratio Juris, 11(22), 73-90.

Daniels, N. (1983). Health care needs and distributive justice. Springer, Boston, MA: In Search of Equity. The Hastings Center Series in Ethics.

Gilli, J. J. (2006). Responsabilidad social. Revista Científica Visión de Futuro, 5, 1-2.

Hammadeh, A. y Mohamad, E. (2016). Social responsibilities of health industry in Abu Dhabi, UAE. Crescent Journal of Medical and Biological Sciences, 3(4), 113-118. 
Kottow, M., y Schramm, F. (2001). Principios bioéticos en salud pública: limitaciones y propuestas. Cuadernos de Saúde Pública, 17, 949-956.

Lozano, J., y Soler, I. (2000). La empresa en la sociedad: responsabilidades éticas. América Latina y España: un mundo compartido, 29-43.

Navarro, V. (1998). Concepto actual de la salud pública. Salud Pública, 49-54.

OMS. (2015). Responsabilidad Social Corporativa. Organización Mundial de la Salud: Organización Panamericana de la Salud. New York. Recuperado de http://www.who.int/es/news-room/27-06-2017-joint-united-nationsstatement-on-ending-discrimination-in-health-care-settings

OMS. (1984). 37a Asamblea Mundial de la Salud, Ginebra, 7 -17 de Mayo de 1984: actas taquigráficas de las sesiones plenarias, informes de las comisiones. Organización Mundial de la Salud. http://www.who.int/iris/ handle/10665/199579

Ortiz, S., y Palavecino, A. (2005). Bioética para una salud pública con responsabilidad social. Acta Bioethica, 11(1).

Pérez, E. V. (2002). Estudio bibliométrico de los artículos originales de la Revista Española de Salud Pública (1991-2000). Parte Primera: indicadores generales. Revista Española de Salud Pública, 76, 659-672.

Rusinque, H., y Constanza, Y. (2016). Importancia de la responsabilidad social en el sector salud. Universidad Militar Nueva Granada.

Sánchez, C., Casabella, J., y Trench, J. (Septiembre, 2009). Propuesta de un sistema de indicadores de responsabilidad social corporativa. En Vintro (Presidencia) Responsabilidad Social XIII Congreso de Ingeniería de Organización, Barcelona.

Soriano Álvarez, C. (2015). The scientific societies and their social responsability in health. Revista de gastroenterología del Perú: órgano oficial de la Sociedad de Gastroenterología del Perú, 293.

Terán, G., Montenegro, C., Gutierrez, E., Tapia, M., Fernández, L., y Rosero, J. (2017). Análisis crítico de la responsabilidad social en entidades de salud. Revista Cubana de Investigaciones Biomédicas, 36-1.

Terris, M. (Junio, 1992). Conceptos sobre promoción de la salud: dualidades en la teoría de la salud pública. En Milton (Presidencia) Discusiones Técnicas sobre Promoción de la Salud. Congreso llevado a cabo en Discusiones Técnicas sobre Promoción de la Salud, Washington, D.C. 
UNESCO. (2005). Declaración universal sobre bioética y derechos humanos. Organización de las Naciones Unidas para la Educación, la Ciencia y la Cultura. Paris. Recuperado de http://portal.unesco.org/es/ev.php-URL_ ID=31058\&URL_DO=DO_TOPIC\&URL_SECTION=201.html

World Business Council. (2017). Measuring Sustainable Lifestyles, Ginebra, ciudad de Suiza. Recuperado de https://www.wbcsd.org/Programs/People/ Sustainable-Lifestyles/Measuring-Sustainable-Lifestyles 


\section{Como citar este artículo}

Gómez, D. (2018). Modelo teórico de indicadores de responsabilidad social en salud. Revista Pensamiento y Acción Interdisciplinaria, 4(1), 8-24. DOI: http://doi.org/10.29035/pai.4.1.8

\section{Dirección de correspondencia}

\section{Danny Amado Gómez Perdomo}

Contador Público. Magíster Scientiarum en Auditoría.

Académico de la Escuela de Administración y Auditoría de la Facultad de Ciencias Sociales y Económicas de la Universidad Católica del Maule, Talca, Chile.

Contacto:

dgomez@ucm.cl

Recibido: 23/06/2018

Aceptado: 05/08/2018 